\title{
The Tiny Ionospheric Photometer: An Instrument for Measuring Ionospheric Gradients for the COSMIC Constellation
}

\author{
Kenneth F. Dymond ${ }^{1, *}$, Jan Bai Nee ${ }^{2}$ and Ronald J. Thomas ${ }^{3}$ \\ (Manuscript received 30 November 1999, in final form 15 January 2000)
}

\begin{abstract}
We describe the specifications for an ultraviolet radiometer for measuring the $\mathrm{F}$-region electron density gradients from the Constellation $\mathrm{Ob}$ serving System for Meteorology, Ionosphere, and Climate (COSMIC). We also present a technique for determining the two-dimensional structure of the ionosphere using the measured ionospheric gradients inferred from observation of the radiative recombination emission at $1356 \AA$ in conjunction with occultations of Global Positioning System satellites observed from the COSMIC satellites. Our scheme uses a nadir viewing UV photometer to characterize the F-region gradients while obtaining altitude information about the F-region from the GPS occultation measurements. We present the results of simulations that demonstrate the applicability and accuracy of the technique and observational concept.
\end{abstract}

(Key words: Ionosphere, Electron density, Remote sensing, Ultraviolet radiometry, Data fusion, Data inversion, GPS occultation)

\section{INTRODUCTION}

The use of total electron content measurements acquired during occultation of the Global Positioning Satellites (GPS) by a receiving platform in low Earth orbit has recently been demonstrated as a viable technique for determining the ionospheric electron density (Hajj et al., 1994). The cornerstone of the technique is use of the Abel inversion to convert the total electron content measurements made by the GPS receiver into electron density profiles. This technique assumes that the ionosphere is spherically symmetric with no horizontal density gradients. The Abel technique works well under certain viewing conditions but its accuracy is generally limited by the spherical symmetry assumption. When there are substantial iono-

\footnotetext{
${ }^{1}$ E. O. Hulburt Center for Space Research, Naval Research Laboratory, Washington, DC, USA

${ }^{2}$ Department of Physics and Chemistry, National Central University, Chungli, Taiwan 320, ROC

${ }^{3}$ Department of Electrical Engineering, New Mexico Institute of Mining and Technology, Socorro, NM 87801, USA

* Corresponding author address: Dr. Kenneth F. Dymond, E. O. Hulburt Center for Space Research, Naval Research Laboratory, Washington, DC 20375-5352, USA; E-mail: dymond@nr1.navy.mil
} 
spheric density gradients present, the Abel inversion technique yields poor results due to an inability to handle the gradients (Hajj et al., 1994). As the GPS occultations occur sporadically around the globe, it is difficult to use them in a systematic way to gather information about the global structure of the ionosphere. Thus, alternative techniques must be used to infer the gradients and to account for them in the inversion process.

Several techniques have been proposed for inferring the ionospheric gradients. On the Constellation Observing System for Meteorology, Ionosphere, and Climate (COSMIC), the GPS occultation measurements will be complemented by ultraviolet radiometric measurements, measurements of the total electron content between the satellites and ground using computerized ionospheric tomography receivers, and ground-based GPS total electron content measurements. The use of UV radiometers provides an accurate measurement of the gradients on a global basis as it does not rely on ground-based receivers.

Naturally occurring ultraviolet and visible emissions from the night sky have been used since the late 1960's as means of determining the densities of electrons and $\mathrm{O}^{+}$ions in the $\mathrm{F}$ region ionosphere. The emissions are produced by radiative recombination of $\mathrm{O}^{+}$ions and electrons to produce atomic oxygen in an excited state that subsequently decays by emitting a photon. These emissions have been observed at visible wavelengths ( 7774 and $8446 \AA$ ) from the ground (Tinsley et al., 1973; Tinsley and Bittencourt, 1975) and at ultraviolet wavelengths (911, 1304, and $1356 \AA$ ) from space (Hicks and Chubb, 1970; Barth and Shaffner, 1970; Chakrabarti et al., 1984; Feldman et al., 1992). The use of the nighttime O emissions to remotely sense the F-region electron density is described by Tinsley and Bittencourt (1975), Chandra et al. (1975), Dymond et al. (1996, 1997), and Meier (1991). The UV emissions are of most interest for space based ionospheric sensing. The Earth's atmosphere absorbs all UV emissions, with wavelengths less than $1800 \AA$, at an altitude of approximately $90 \mathrm{~km}$. Thus, there is no UV background. The Earth's atmosphere is transparent to the visible emissions and, therefore, there is a visible background due to city lights, forest fires, thunderstorms, noctilucent clouds, and reflection of moon light; these background emissions would require measurement and subtraction from the nightglow signal. This background subtraction can lead to large uncertainties in the derived airglow signal. The problems caused by background emission are far worse on the dayside because the background emissions can originate above $100 \mathrm{~km}$.

The radiative recombination emissions cannot be used for ionospheric sensing on the dayside, even though the emissions are always present. The $\mathrm{O}$ emissions, with the exception of the $911 \AA$ emission, are excited by photoelectron impact on the dayside, which renders them insensitive to the ionospheric state. The $911 \AA$ emission could, in principle, be used for daytime ionospheric sensing, as it is not affected by photoelectron impact. However, during the daytime, the spectral region lying near the $911 \AA$ emission is contaminated by emission from neutral and ionized nitrogen; this effectively renders the $911 \AA$ measurements useless for daytime ionospheric sensing.

We present the results of a study to demonstrate the use of UV radiometry with GPS occultations for the COSMIC program. The COSMIC program is being built by the National Space Program Office (NSPO) of the Republic of China (Taiwan), the University Consortium for Atmospheric Research (UCAR), the Jet Propulsion Laboratory, and the Naval Research 
Laboratory. We describe the results of a design study undertaken to specify the desirable characteristics of an ultraviolet radiometer capable of accurately measuring the nighttime ionospheric gradients. We also describe an algorithm that can be used to simultaneously invert the UV nadir data and the GPS occultation data. This algorithm can accurately retrieve the electron density distribution over the region where the occultation occurs regardless of the gradients.

\section{RADIATIVE RECOMBINATION PROCESS}

There are several candidate emissions that can be used to determine the electron density in the nighttime ionosphere. These emissions occur at 911,1304 , and $1356 \AA$. The $911 \AA$ is the easiest of these three emissions to interpret. The emission is optically thin and therefore no radiative transport modeling is required. The emission is produced solely by radiative recombination. The $1356 \AA$ emission is the result of a spin forbidden transition in atomic oxygen and as such is largely optically thin. However, although the transition is forbidden, the large abundance of atomic oxygen in the earth's thermosphere causes the optical depth for resonant scattering of this emission to reach unity near $100 \mathrm{~km}$, which requires some radiative trransport modeling for accurate interpretation. The $1304 \AA$ emission is produced by an allowed transition in atomic oxygen. Thus, this emission is optically thick with a vertical optical depth exceeding several thousands near $100 \mathrm{~km}$. Interpretation of the $1304 \AA$ requires extensive radiation transport modeling. This makes it difficult to use for ionospheric sensing. In addition to the radiative recombination process, the 1304 and $1356 \AA$ emissions can also be produced by neutralization of $\mathrm{O}^{+}$by $\mathrm{O}^{-}$. This complicates the use of these emissions as the density of $\mathrm{O}^{-}$is related to the density of $\mathrm{O}$. We have found that this complication is not important as the $\mathrm{O}$ density gradients are much smaller than the $\mathrm{O}^{+}$gradients. Thus, an algorithm that uses the gradient in the UV intensity rather than the intensity is relatively insensitive to the $\mathrm{O}$ density gradient, as is presented below.

The $911 \AA$ emission is easily modeled and interpreted. The volume emission rate for the emission is proportional to the product of the electron and ion densities. In the nighttime Fregion ionosphere, the electron and $\mathrm{O}^{+}$densities are nearly equal. The vertical UV radiance (Feldman et al., 1992), $I_{9 \mid 1}$, is given by:

$$
I_{911}=10^{-6} \alpha_{911} \int_{0}^{z_{5}} n_{e}(z) n_{0^{+}}(z) d z,
$$

where $\mathrm{n}_{\mathrm{e}}$ is the electron density at altitude $z, n_{0^{+}}$is the $\mathrm{O}^{+}$density at $z, d z$ is the differential path length from the observer, $Z_{s}$ is the satellite altitude, and $\alpha_{911}$ is the radiative recombination rate, which is $3.5 \times 10^{-13} \mathrm{~cm}^{3} \mathrm{~s}^{-1}$ at $1160 \mathrm{~K}$ (Melendez-Alvira et al., 1999). The constant, $10^{-6}$, converts the radiance from units of photons $\mathrm{cm}^{-2} \mathrm{~s}^{-1}$ to Rayleighs. (A Rayleigh is the number of photons emitted per second into $4 \pi$ steradians by a column with a $1 \mathrm{~cm}^{2}$ cross-section expressed in megaphotons; typical airglow radiances range between 0.1 and $10^{5}$ Rayleighs.) We assume that the ionospheric electron temperature is isothermal at $1160 \mathrm{~K}$. This assumption is rarely valid, but we have found that it does not affect the retrievals. Equation (1) can be rewritten to express the overall radiance in terms of the peak electron density (and hence the 
peak $\mathrm{O}^{+}$density by charge neurality) in the F-region, $n m F 2$. Thus the retrieved $n m F 2$ is proportional to the square root of the intensity $I_{911}$ and is inversely proportional to the radiative recombination rate coefficient. Since the radiative recombination rate coefficient is proportional to $T e^{-1 / 2}$, the retrieved $n m F 2$ scales as $T e^{1 / 4}$, which is a very weak dependence on the electron temperature.

The $1356 \AA$ emission is slightly more complicated than the $911 \AA$ emission. The emission shows mild opacity effects due to multiple scattering of the radiation by atomic oxygen in the thermosphere. The emission is also a doublet emission originating in a singlet upper state with wavelengths 1356 and $1358 \AA$. The two lines have slightly different opacities that must be included in the model. The volume emission rate, $\varepsilon_{0}(z)$, for the emission is given by (Tinsley and Bittencourt, 1975):

$$
\varepsilon_{0}(z)=\gamma \beta_{1356} \frac{\kappa_{1} \kappa_{2} n_{e}(z) n_{O}(z) n_{O^{+}}(z)}{\kappa_{2} n_{O^{+}}(z)+\kappa_{3} n_{O}(z)}+\gamma \alpha_{1356} n_{e}(z) n_{O^{+}}(z),
$$

where $\gamma$ is the branching ratio for the 1356 or $1358 \AA$ line $(0.791$ and 0.209 , respectively, (Meier, 1991)), $\alpha_{1356}$ is the radiative recombination rate $7.5 \times 10^{-13} \mathrm{~cm}^{-3} \mathrm{~s}^{-1}$ (Melendez-Alvira et al., 1999), $\beta_{1356}$ is the fraction of neutralizations that produce atoms in the ${ }^{5} \mathrm{~S}$ state, 0.54 , and the coefficients $\kappa_{1} \kappa_{2}$, and $\kappa_{3}$ are $1.3 \times 10^{-15}, 1 . \times 10^{-7}$, and $1.4 \times 10^{-10}$, respectively, all in units of $\mathrm{cm}^{3} \mathrm{~s}^{-1}$ (Tinsley and Bittencourt, 1975). The relevant reactions and their rates summarized in Table 1 . To calculate the volume emission rate including multiple scattering, $\varepsilon(z)$, the following integral equation is solved (Strickland and Rees, 1974; Strickland, 1979; Anderson and Meier, 1985; and Meier 1991):

$$
\varepsilon(z)=\varepsilon_{0}(z)+n_{0}(z) \sigma \int_{z_{\min }}^{z_{\max }} \varepsilon\left(z^{\prime}\right) H\left(\left|\tau(z)-\tau\left(z^{\prime}\right)^{\prime}\right| t(z)-t\left(z^{\prime}\right) \mid\right) d z^{\prime}
$$

where $\sigma$ is the line-center scattering cross-section $\left(2.499 \times 10^{-18} \mathrm{~cm}^{2}\right.$ and $1.242 \times 10^{-18} \mathrm{~cm}^{2}$ for the 1356 and $1358 \AA$ lines, respectively (Meier, 1991)), $\tau$ is the vertical resonant scattering optical depth, $t$ is the vertical pure absorption optical depth, and $H$ is the Holstein function. The vertical optical depths for scattering and pure absorption are given by:

$$
\tau(z)=\sigma \int_{z}^{\infty} n_{o}\left(z^{\prime}\right) d z^{\prime}
$$

and

$$
t(z)=\sigma_{\bullet_{2}}^{a b s} \int^{\infty} n_{o}\left(z^{\prime}\right) d z^{\prime},
$$

where $\sigma_{o_{2}}^{a b s}$ is the absorption cross-section for $\mathrm{O}_{2}\left(7.6 \times 10^{-18} \mathrm{~cm}^{2}\right.$ and $8.0 \times 10^{-18} \mathrm{~cm}^{2}$ for the 1356 and $1358 \AA$ lines, respectively, at $1160 \mathrm{~K}$ taken from Wang et al., (1987)) and $n_{o_{2}}$ is the density of $\mathrm{O}_{2}$, the only absorber of the $1356 \AA$ radiation. The Holstein $H$ function is given by:

$$
H(\tau, t)=\frac{1}{2 \sqrt{\pi}} \int_{-\infty}^{\infty} e^{-2 x^{2}} E_{1}\left(\tau e^{-x^{2}}+t\right) d x,
$$

where $x$ is the photon's frequency, $e^{-x^{2}}$ is the Gaussian line shape appropriate to a Doppler 
Table 1. This table contains the reactions rate coefficients relevant to the ionospheric nightglow processes. The first four rows pertain to the $1356 \AA$ emission. The last row pertains to the 911 emission. Te indicates the electron temperature in Kelvins.

\begin{tabular}{|c|c|c|}
\hline Reaction & $\overline{\text { Rate Coefficient }}$ & $\overline{\text { Reference }}$ \\
\hline $\begin{array}{l}\mathrm{O}^{+}+e \\
\rightarrow \mathrm{O}+h v(1356 \AA)\end{array}$ & $\alpha_{1356}=7.3 \times 10^{-13}\left(1160 / \mathrm{T}_{\mathrm{e}}\right)^{-1 / 2} \mathrm{~cm}^{3} \mathrm{~s}^{-1}$ & $\begin{array}{c}\text { Melendez-Alvira et al } \text {. } \\
\text { [1999] }\end{array}$ \\
\hline $\mathrm{O}+e \rightarrow \mathrm{O}^{-}+h v$ & $k_{1}=1.3 \times 10^{-13} \mathrm{~cm}^{3} \mathrm{~s}^{-1}$ & $\begin{array}{c}\text { Melendez-Alvira et al. } \\
\text { [1999] }\end{array}$ \\
\hline $\begin{array}{c}\mathrm{O}^{-}+\mathrm{O}^{+} \rightarrow \mathrm{O}^{*}+\mathrm{O} \\
(1356 \AA)\end{array}$ & $\begin{array}{l}k_{2}=1.0 \times 10^{-1} \mathrm{~cm}^{3} \mathrm{~s}^{-1} \\
\beta_{1356}=0.54\end{array}$ & $\begin{array}{c}\text { Tinsley and Bittencourt } \\
\text { [1975] }\end{array}$ \\
\hline $\mathrm{O}+\mathrm{O}^{-} \rightarrow \mathrm{O}_{2}+e$ & $k_{3}=1.4 \times 10^{-10} \mathrm{~cm}^{3} \mathrm{~s}^{-1}$ & $\begin{array}{c}\text { Tinsley and Bittencourt } \\
\text { [1975] }\end{array}$ \\
\hline $\begin{array}{c}\mathrm{O}^{+}+e \\
\rightarrow \mathrm{O}+h v(911 \AA)\end{array}$ & $\alpha_{911}=3.5 \times 10^{-13}\left(1160 / T_{c}\right)^{-1 / 2} \mathrm{~cm}^{3} \mathrm{~s}^{-1}$ & $\begin{array}{c}\text { Melendez-Alvira et al } \text {. } \\
\text { [1999] }\end{array}$ \\
\hline
\end{tabular}

broadened spectral line, and $E_{1}(x)$ is the exponential integral of the first kind (Abramowitz and Stegun, 1972). The $H$ function is proportional to the probability that a photon will propagate from region $z^{\prime}, z^{\prime}+d z^{\prime}$ to the region $z, z+d z$.

A variety of approaches have been used to solve the integral equation, 3 . In this work, the integral equation has been solved by $\Lambda$ iteration (Duderstadt and Martin, 1979). In this scheme, the iteration is started using $\varepsilon_{0}(z)$ as the first guess at the solution. The new scattered volume emission rate is calculated and substituted into the integral, which is then evaluated. The iteration process is stopped when the volume emission rate changes by less than $0.01 \%$ between iterations. A typical set of volume emission rates for the $911 \AA$ and $1356 \AA$ emissions are included in Fig. 1. After the volume emission rate has been calculated, the column intensities must be calculated by evaluating:

$$
I_{1356}=10^{-6} \sum \int_{0}^{z_{s}} T\left(\left|\tau(z)-\tau\left(Z_{s}\right)\right|\right),\left(\left|t(z)-t\left(Z_{s}\right)\right|\right) \varepsilon(z) d z,
$$

where the sum is over the 1356 and $1358 \AA$ lines. The transmission function $T$ is given by: 


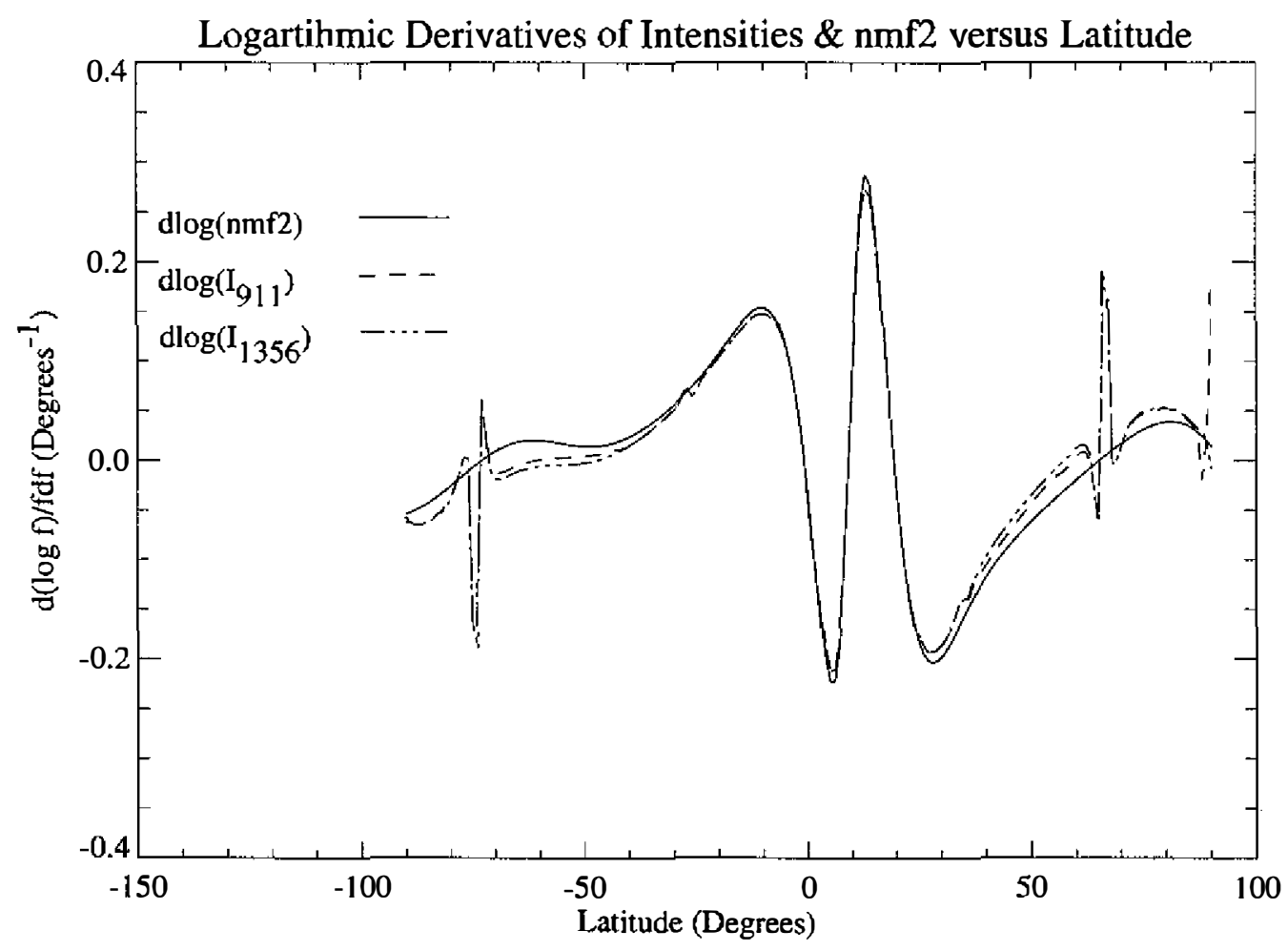

Fig. 1. This figure shows the gradients in the $1356 \AA$ and $911 \AA$ radiances and the twice the $n m F 2$ gradient ( 911 and $1356 \AA$ gradients are proportional to $\left.(n m F 2)^{2}\right)$. The logarithmic derivatives are plotted as these remove the dependencies on the radiative recombination rate coefficients. Note how closely both the gradients in the 911 and $1356 \AA$ radiances track the $n m F 2$ gradient. Differences are attributed to the variation of the scale height, peak height of the layer, and the electron temperature. The spikes in the gradients at high latitudes are due to the IRI-90 model and will not be present in the ionosphere.

$$
T(\tau, t)=\frac{1}{\sqrt{\pi}} \int_{-\infty}^{\infty} e^{-x^{2}} \exp \left(-\tau e^{-x^{2}}+t\right) d x,
$$

where $x$ is the photon's frequency. The $\mathrm{O}$ density has entered the problem in three ways: it has contributed to the volume emission rate through the $\mathrm{O}^{+}-\mathrm{O}^{-}$neutralization; it has enhanced the volume emission rate through multiple scattering; and it has scattered photons out of the fieldof-view through the transmission function.

The radiation transport contribution to the $1356 \AA$ radiance is generally small. The scatterer of the $1356 \AA$ radiation is $\mathrm{O}$ and the $\mathrm{O}$ density peaks below $150 \mathrm{~km}$. The ionospheric $\mathrm{O}^{+}$ and electron densities generally peak above $300 \mathrm{~km}$ at night. This is due to the overall collapse 
of the ionosphere at night due to recombination processes. At night, the $\mathrm{O}^{+}$charge exchanges with neutral molecules to produce molecular ions. The subsequent recombination of those molecular ions rapidly eats away the bottomside of the electron density profile (Chamberlain and Hunten, 1987); this reduces the overlap of the ionosphere with the O layer. This, in turm, reduces the amount of resonant scattering the $1356 \AA$ photons undergo before they escape to space. Therefore, reduced scattering means less entrapment of the $1356 \AA$ photons and an overall decrease in the $1356 \AA$ emission intensity. However, the entrapment of the $1356 \AA$ photons by resonant scattering increases the emission intensity by at most $5 \%$. We have included it in our modeling because the UV radiometer for the COSMIC program should be able to measure $1356 \AA$ radiance with less than $1 \%$ uncertainty over a wide range of ionospheric conditions.

The typical radiances seen in the nighttime ionosphere at either 1356 or $911 \AA$ drive the design of the UV instrument. These radiances were simulated using the International Reference Ionosphere (IRI-90) to provide the electron and $\mathrm{O}^{+}$densities and the electron temperature. The Mass Spectrometer and Incoherent Scatter model (MSIS-86: Hedin, 1987) was used to produce the $\mathrm{O}$ densities used in the calculation of the neutralization component at $1356 \AA$. The radiances were estimated for the minimum of the 11 year solar cycle as the ionospheric densities, and hence the radiances, are lowest at that time. The minimum radiances were of order 0.1 Rayleigh at $911 \AA$ and 0.2 Rayleigh at $1356 \AA$.

\section{INSTRUMENT}

The UV instrument for the COSMIC program has to provide precise and accurate measurements of the radiance in a compact, low power package. The simplest and most sensitive option for the COSMIC was the use of a narrow band radiometer. The most difficult part of the design was the selection of a filter. Filter technology is not as advanced in the vacuum UV as it is in the visible and infrared. Narrow band filters with a compact design are not yet commercially available. At $911 \AA$, the instrument's passband is determined by the choice of a thin metal film filter and photocathode combination. At $1356 \AA$, a similar choice is possible using a window that blocks short wavelengths and a photocathode to eliminate long wavelength sensitivity. We considered two design options. The first option was to use the $911 \AA$ emission. The second option was to use the $1356 \AA$ emission. Both options assumed that the instrument would consist of an off-axis parabolic telescope with a photon counting detector and electronics.

An instrument's sensitivity is defined as the number of photons detected per second at an input radiance of 1 Rayleigh:

$$
S=\frac{10^{6}}{4 \pi} Q T R A \Omega,
$$

where $Q$ is the quantum efficiency of the photocathode on the detector (number of photons detected / number of photons incident), $T$ is the filter transmittance, $R$ is the reflectance of the telescope, $A$ is the collecting area of the telescope in $\mathrm{cm}^{2}$, and $\Omega$ is the instrument's field-ofview in steradians. The factor $10^{6 / 4} \pi$ converts the radiance from units of Rayleighs to pho- 
tons $\mathrm{s}^{-1} \mathrm{~cm}^{-2}$ steradian $^{-1}$ (the $4 \pi$ factor is required here to convert from the omnidirectional radiance, expressed in Rayleighs, to a specific radiance per steradian). We selected a field-ofview $\Omega$ based on the desired spatial resolution for determining the ionospheric gradients. The field-of-view that was selected is $3^{\circ} \times 15^{\circ}$ (along the orbit track $\times$ across the satellite's ground track). This field-of-view results in a spatial coverage of approximately $20 \mathrm{~km} \times 100$ $\mathrm{km}$ when projected to $350 \mathrm{~km}$, the average height of the F-region peak, when viewed from a satellite at $750 \mathrm{~km}$. The $23 \mathrm{~km}$ resolution is smeared out to $30 \mathrm{~km}$ when the integration time of the instrument ( 1 second) and the vehicle's speed are included. The instrument's field-ofview, $\Omega$, is then approximately 0.014 steradians. We have assumed a $5 \mathrm{~cm}$ diameter for the collecting optic; thus, the collecting area is $19.6 \mathrm{~cm}^{2}$.

We now estimate the efficiency of a radiometer operating at $911 \AA$. The detector we chose for this estimate is a channeltron. The quantum efficiency of a channeltron at $911 \AA$ is about $10 \%$. The channeltron's quantum efficiency drops by roughly an order of magnitude to $1 \%$ at $1216 \AA$. The transmittance of an indium thin film has been measured at the Naval Research Laboratory and found to be $3 \%$ at $911 \AA$ and $0.5 \%$ at $1216 \AA$ (G. R. Carruthers, private communication, 1996). Silicon carbide has the highest reflectivity at $911 \AA$ of any substance. Its reflectance has been measured at NRL and found to be $35 \%$; this reflectance increases slightly at $1216 \AA$, but the difference is of little consequence here. When we substitute these numbers into equation (9), we calculate the sensitivity at $911 \AA$ to be 23 counts s-1 Rayleigh $^{-1}$ and 0.35 count s$^{-1}$ Rayleigh $^{-1}$ at $1216 \AA$. Thus, we find that an instrument will detect 2.3 counts $\mathrm{s}^{-1}$ at $911 \AA$ at our desired minimum signal of 0.1 Rayleighs. However, the bright $1216 \AA$ (Hydrogen Lyman- $\alpha$ ) emission can be fairly bright even at night. The $1216 \AA$ is scattered over from the dayside to the nightside by atomic hydrogen in the Earth's geocorona. Meier (1991) gives a nighttime radiance of approximately 1700 Rayleighs for Lyman- $\alpha$. This means that the $911 \AA$ radiometer will see 600 counts s$^{-1}$ due to Lyman- $\alpha$ with a 2.3 count $\mathrm{s}^{-1}$ due to $911 \AA$ superimposed. Clearly, this is not a desirable situation.

We now estimate the efficiency of a radiometer operating at $1356 \AA$. The detector we chose for this estimate is a photomultiplier tube with a cesium iodide photocathode and magnesium fluoride window. The quantum efficiency of a photomultiplier of this type at $1356 \AA$ is about $10 \%$. We have opted to block the bright H I $1216 \AA$ emission and the O I $1304 \AA$ emission by using a heated strontium fluoride filter. The short wavelength transmission cutoff for strontium fluoride occurs at $1280 \AA$ at a temperature of $25^{\circ} \mathrm{C}$. The transmittance of a $5 \mathrm{~mm}$ thick strontium fluoride filter has been measured at a temperature of $80^{\circ} \mathrm{C}$ by the NRL. The transmittance was $40 \%$ at $1356 \AA$, less than $4 \%$ at $1304 \AA$, and $0 \%$ at $1216 \AA$. The reflectance of a magnesium fluoride over aluminum coating for the telescope mirror at $1356 \AA$ is about $80 \%$ (Samson, 1967). When these numbers are substituted into equation (10), we calculate the sensitivity at $1356 \AA$ to be 700 counts s $^{-1}$ Rayleigh $^{-1}$ and less than 70 count s $^{-1}$ Rayleigh ' at $1304 \AA$. Thus, we find that an instrument will detect 140 counts s $^{-1}$ at $1356 \AA$ at our desired minimum signal of 0.2 Rayleighs. However, the signal is slightly contaminated by the $\mathrm{O} I$ $1304 \AA$ emission. As this emission is also produced by radiative recombination, the contamination to the $1356 \AA$ signal by the $1304 \AA$ emission is deemed acceptable. Thus, the $1356 \AA$ radiometer produces a higher quality measurement than the $911 \AA$ radiometer does.

The primary difficulty with the 1356 emission measurements is the contribution to the 
signal by $\mathrm{O}^{+}-\mathrm{O}$ neutralization. Since neutralization component is related to the $\mathrm{O}$ density, the neutralization contribution to the ionospheric gradients inferred from the $1356 \AA$ emission was examined. The nadir radiances for the $911 \AA$ and $1356 \AA$ emissions were simulated using the International Reference Ionosphere (IRI-90: Bilitza, 1990) for the $\mathrm{O}^{+}$and electron densities and the electron temperature. The $\mathrm{O}$ density was taken from the Mass Spectrometer and Incoherent Scatter model (MSIS-86: Hedin, 1987) and used to calculate the $\mathrm{O}^{+}-\mathrm{O}^{-}$neutralization contribution to the volume emission rate at $1356 \AA$. To compare the gradients without having to scale results to remove the effects of the different radiative recombination rate coefficients, the logarithmic derivatives are used. Figure 1 shows the comparison of the $n m F 2$ variation and the 1356 and $911 \AA$ gradients. The $1356 \AA$ logarithmic derivative closely tracks the $n m F 2$ logarithmic derivative indicating that the neutralization component does not significantly affect the $1356 \AA$ gradient measurements. Furthermore, this Figure suggests that gradient of the radiometer data should be used in an algorithm to invert the GPS occultation data and correct for the gradients.

The COSMIC photometer will operate at $1356 \AA$. This is based on the accuracy of the gradients as seen in Fig. 1, on the strong contamination of the $911 \AA$ signal by Lyman- $\alpha$, and the higher sensitivity possible at $1356 \AA$.

\section{ALGORITHM}

\section{1 Algorithm Introduction}

The use of UV radiometric data to correct GPS occultation data for ionospheric density gradients has been simulated. We developed and tested an algorithm that jointly inverts both types of data to produce a high fidelity ionospheric reconstruction along the orbit track. The GPS total electron data are in the form of an occultation or "limb scans" and therefore the GPS data provides high quality altitude information. However, the GPS data are integrated electron densities along the line-of-sight to the GPS satellite and therefore may be affected by gradients along the line-of-sight. The intensity measured by the radiometer is proportional to the vertical column integral of the square of the electron density and, therefore, contains no altitude information. But, the radiometer data contains high quality gradient information. The use of the two data sets together offsets the weaknesses in each data set while taking advantage of their strengths. In this work, we will assume that the photometer images the $911 \AA$ emission, which tracks the $n m F 2$ gradients as well as the $1356 \AA$ radiance does.

The GPS receiver measures the total electron content given by:

$$
T E C(\varphi)=\int_{0}^{\infty} n_{e}(z(s)) d s,
$$

where $n_{e}$ is the electron density at altitude $z$ and $d s$ is the differential path length from the observer. The derivation of the radiometer radiances is described above.

The altitude distribution of the $\mathrm{O}^{+}$and electron density was parameterized using a generalized Chapman layer (Chamberlain and Hunten, 1987). We assume that the electron and $\mathrm{O}^{+}$ densities are equal, which is a very good approximation below the $\mathrm{H}^{+} / \mathrm{O}^{+}$transition height in 
the nighttime F-region. We used five parameters to characterize the ionosphere: the altitude where the density peaks, $h m F 2$; the density at the peak, $n m F 2$; and three parameters to characterize the scale height, $\mathrm{H}(\mathrm{z})$, which is one-half the plasma scale height for the form of the Chapman layer given below. The Chapman function for describing the $\mathrm{O}^{+}$density, $n_{o^{+}}(z)$, is:

$$
n_{o^{+}}(z)=h m F 2 \operatorname{ex}\left[\left\{\frac{1}{2}\left[1-\frac{z-h m F 2}{H(z)}-\exp \left(-\frac{z-h m F 2}{H(z)}\right)\right]\right\},\right.
$$

where $z$ is the altitude. The scale height is assumed to be a constant below $h m F 2$ and to vary quadratically with the difference in altitude above the peak: $H(z)=H_{0}+H_{1}(z-h m F 2)+H_{2}(z-$ $h m F 2)^{2}$, where $H_{0}, H_{1}$, and $H_{2}$ are parameters. This form of the Chapman layer is an extension to the linearly varying scale height proposed by Picone et al. (1997) and is a reasonable approximation for describing the ionospheric density.

The along-track variation of the electron is parameterized by placing Chapman profiles spaced every degree along the orbit track. The profiles are all uncoupled and no smoothing or regularization is used; this causes the inversion to be more sensitive to point to point variations in the photometer data. These point-to-point variations are due to photon shot noise added to the simulated along-track radiance. The same altitude variation is assumed for all of the profiles, as the altitude variation is determined at one location near the tangent point locus of the GPS occultation. The individual profiles are scaled by $n m F 2$ to characterize the along track electron density variations. The $n m F 2$ variation between the profiles is given by a quadratic Lagrange interpolation polynomial (Press et al., 1992). These are reasonable approximations to make as the GPS occultations provide accurate altitude distribution information while the photometer data provide accurate information on $n m F 2$ and no information about the altitude distribution. However, both the scale height and peak height of the electrons are known to vary with latitude in the ionosphere. The deficiencies in this approximation will be discussed later.

The line-of-sight of a GPS occultation spans $56^{\circ}$ degrees of latitude from a satellite at an altitude of $750 \mathrm{~km}$ when the tangent altitude of the line-of-sight is $100 \mathrm{~km}$. Thus, our model has 56 parameters to describe the along-track $n m F 2$ variation and 4 additional parameters to describe the shape of the profiles.

The inversion algorithm uses an iterative approach, based on Discrete Inverse Theory (Menke, 1989), to seek the maximum likelihood estimate (minimum of the chi-squared statistic) of the ionospheric parameters based on the fit of the model to the data. The DIT approach starts by estimating the "data" based on the current parameter values. The $\mathrm{O}^{+}$densities are used to calculate the intensity seen by the photometer and the total electron content measured by the GPS receiver. The $\chi^{2}$ statistic (Bevington, 1969) is then calculated and used to compare the model to the "data". If the fit is deemed to be acceptable, the algorithm terminates and calculates the "best fit" ionosphere; otherwise, new values for the model parameters are chosen and the whole process is repeated. A fit is considered to be acceptable when the $\chi^{2}$ changes by less than $0.1 \%$ between steps. Since the radiative recombination emissions are proportional to the square of the electron density, the algorithm is non-linear. The parameters are iteratively adjusted using the Levenberg-Marquardt scheme (Press et al., 1992) to seek the 
minimum $\chi^{2}$. In addition to the ionospheric parameters that obtained the "best fit", the uncertainties in those parameters are also estimated and outputted. Errors in the retrieved densities are calculated from the best fit parameters and their uncertainties using conventional error propagation techniques (Bevington, 1969).

\section{2 "Data" Simulation}

Evaluations of the algorithm's performance against "realistic data" were made using the IRI-90 (Bilitza, 1990) to generate the "data" which were inverted using the algorithm. IRI-90 was run for a set of solar activities and geomagnetic conditions characteristic of the maximum of the 11 year solar cycle, which should be applicable to the COSMIC program (expected launch 2003). The "data" were simulated using the electron temperature to determine the appropriate recombination rate coefficient and the $\mathrm{O}^{+}$and electron densities to calculate the $911 \AA$ intensities and total electron content.

Uncertainties in the "data" were generated by assuming that the photometer "data" are shot noise limited (uncertainty equal to the square root of the number of counts) and that the TEC data had a random variance of 0.003 TECU (Hajj et al., 1994). (A TECU or total electron content unit is defined to be $10^{16}$ electrons $\mathrm{m}^{-2}$. The nighttime limb viewing TEC is expected to be on the order of $100 \mathrm{TECU}$, during the GPS occultation measurements made by the COSMIC GPS receiver.) The shot noise limit assumption is valid because the photometers count photons and have negligible dark count rates. The synthetic photometer "data" without noise were converted from units of Rayleighs, to units of counts per bin per second by applying an intensity scaling factor (100 count second ${ }^{-1}$ Rayleigh $^{-1}$, which is typical of the photometers being designed for the COSMIC program) and multiplying by the integration time (10 seconds). These uncertainties were then superimposed on the "data" by generating Poisson random deviates. New uncertainties, which accurately represent the "noisy photometer data", were derived by taking the square root of the noisy count rate "data". The "noisy data" and their error bars were then converted back to intensities in Rayleighs. A similar approach was used for the TEC data; however, Gaussian random deviates were used instead of Poisson deviates. These synthetic "data" and their uncertainties were used in the inversion.

We have assumed that the vehicle is at $750 \mathrm{~km}$ altitude in a polar orbit and the GPS occultations are occurring in the orbit track. This gives us a reasonable geometry with which to test the algorithm and maximizes the effects of ionospheric gradients.

\section{3 Algorithm Test Results}

Figure 2 shows the instrument viewing geometry though the IRI-90 ionosphere used in the simulation. The curved lines across the image represent every $10^{\text {th }}$ GPS occultation ray path and the vertical lines represent every $10^{\text {th }}$ photometer exposure. The dashed curve in the figure shows the trajectory of the tangent ray point through the ionosphere. This particular simulation occurs in a region with strong electron density gradients. The solid line Fig. 3a shows the GPS TEC "data", while the dashed line shows the fit using our algorithm. The solid line in Fig. 3b shows the photometer "data", while the dashed line shows the fit to the "data". The algorithm accurately fits both sets of "data". Figure 4a shows a comparison of the IRI-90 


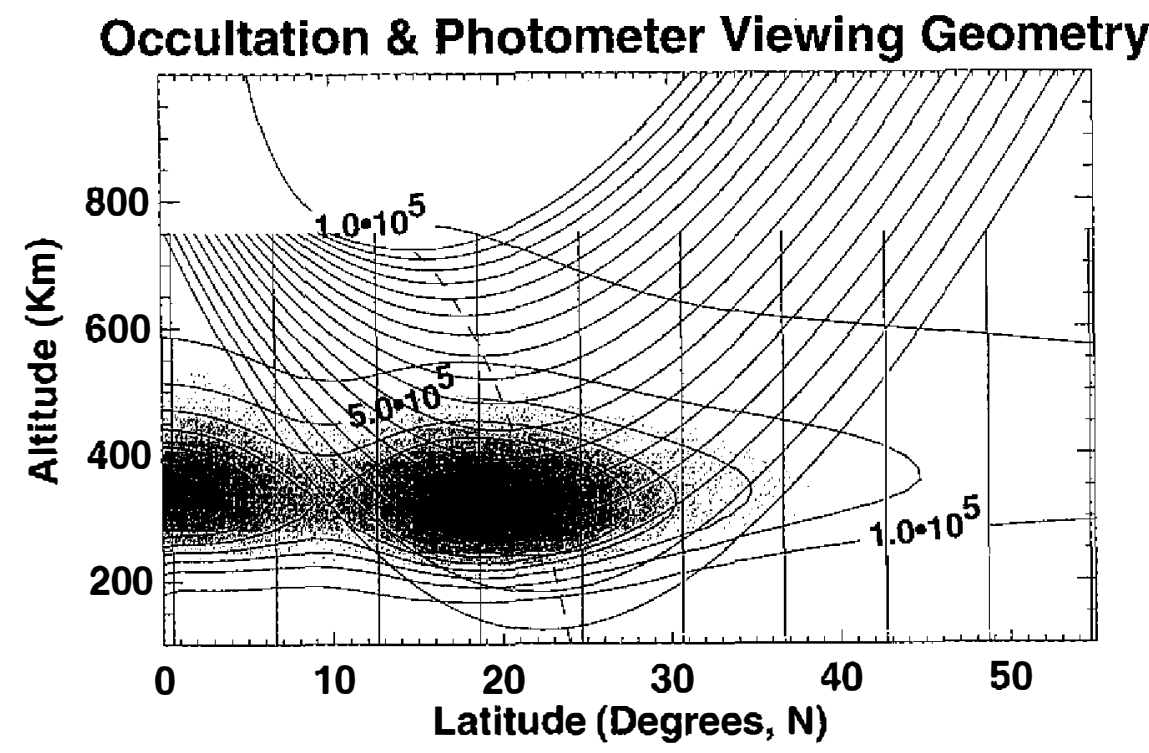

Fig. 2. This is the instrument viewing geometry though the IRI-90 ionosphere used in the simulation. The curved lines across the image represent every $10^{\text {th }}$ GPS occultation ray path and the vertical lines represent every $10^{\text {th }}$ photometer exposure. The dashed curve in the figure shows the trajectory of the tangent ray point through the ionosphere.

density along the tangent ray trajectory (solid line) to the two-dimensional algorithm fit (dashed line), the Abel inversion retrieval (dash-dot), and a discrete inverse theory fit assuming spherical symmetry and our modified Chapman layer parameterization (dash-triple dot). Note that the two-dimensional algorithm does a much better job of retrieving the density than either technique that relies on the spherical symmetry assumption. Panel $4 \mathrm{~b}$ shows the fractional ((2D - IRI-90) / IRI-90) error between the two-dimensional retrieval and the IRI-90 ionosphere. A large island of low retrieval error (less than $15 \%$ ) is seen to exist from roughly $10^{\circ}$ to $30^{\circ}$ latitude. In this figure, negative values denote that the two-dimensional retrieval is below the IRI-90 ionosphere. Figures $4 \mathrm{c}$ and $4 \mathrm{~d}$ show the IRI-90 ionosphere and the retrieved ionosphere, respectively. Note that the ionospheric peak height does not change with latitude in the retrieval, while it does in the IRI-90 ionosphere. This is caused by the assumption, in the two-dimensional algorithm, that the altitude distribution of the electrons does not change with latitude. This assumption causes the "tongue" of enhanced electron density evident in Fig. 3d above $20^{\circ}$ latitude. It also results in the topside scale height being overestimated by the twodimensional algorithm, as seen in Fig. 4a.

\section{CONCLUDING REMARKS}

We have presented the ionospheric physics relevant to making nighttime measurements of the density gradients in the F-region ionosphere. Then we described the Tiny Ionospheric 
GPS TEC

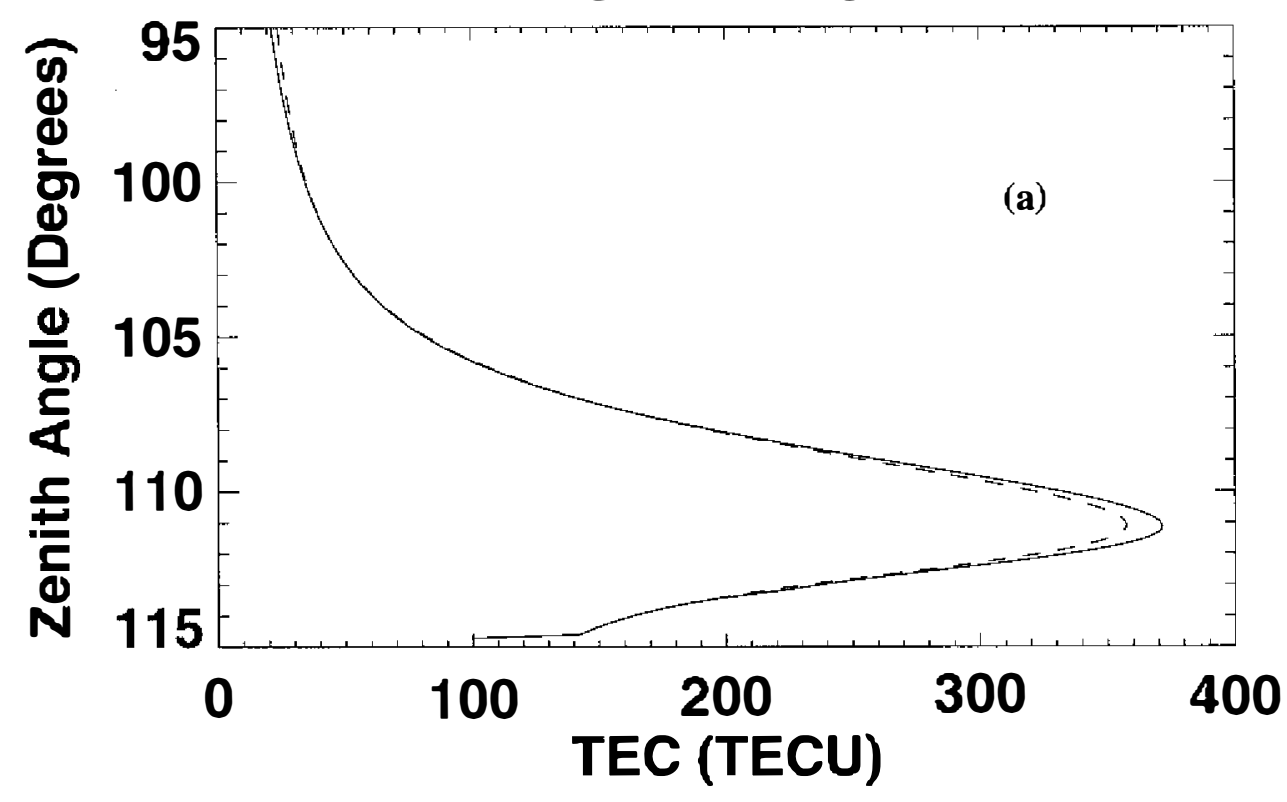

Photometer Radiance

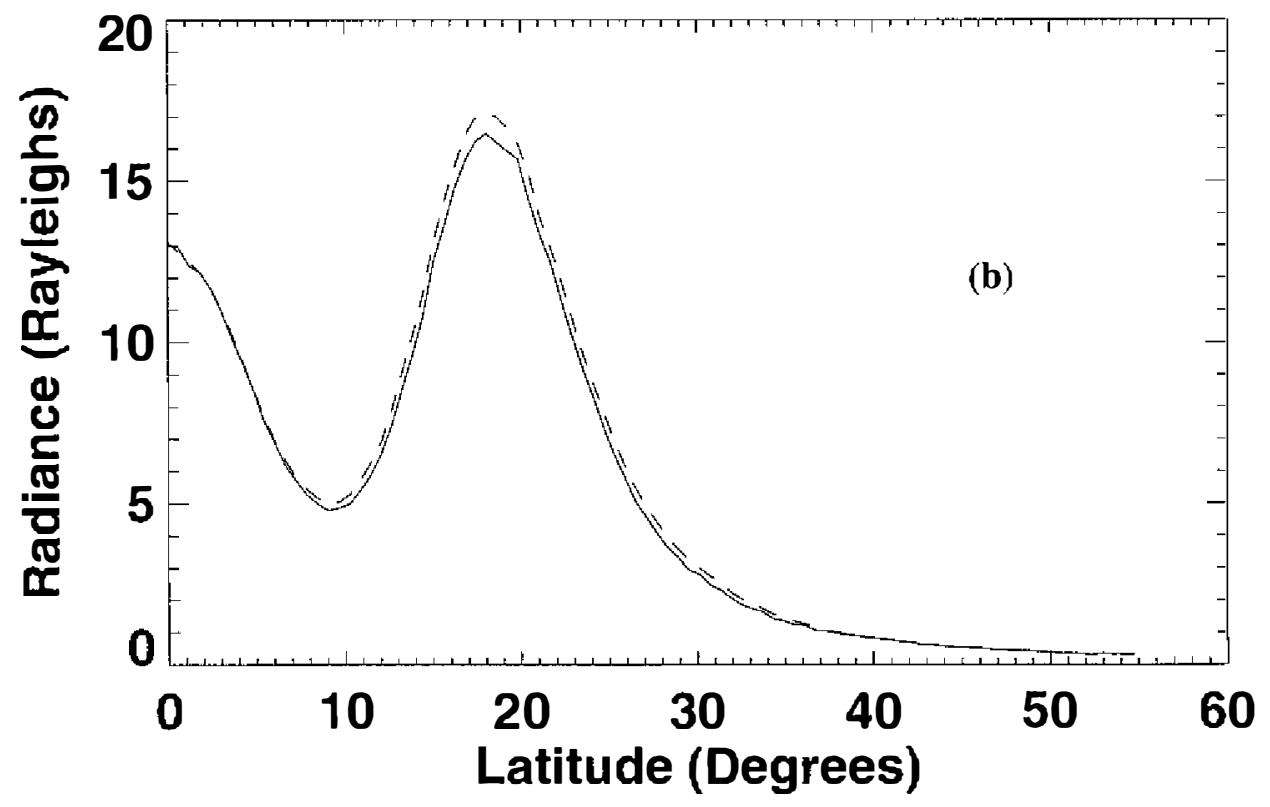

Fig. 3. The solid line in (a) shows the GPS TEC "data", while the dashed line shows the fit using our algorithm. The solid line in (b) shows the photometer "data", while the dashed line shows the fit to the "data" 
Photometer (TIP) and the considerations that went into its design. The instrument will be a radiometer operating at $1356 \AA$. It is expected to have a sensitivity of approximately 700 counts $\mathrm{S}^{-1}$ Rayleigh $^{-1}$. For comparison, the Special Sensor Ultraviolet Limb Imagers for the United States Air Force's Defense Meteorological Satellite Program (DMSP) satellites have sensitivities at $1356 \AA$ ranging from $0.5-1$ counts s $^{-1}$ Rayleigh $^{-1}$.

We have presented an algorithm for simultaneously inverting nighttime nadir radiometer data with GPS occultation data. Our approach is to use a simple two-dimensional parameterization of the ionospheric electron density and to iteratively adjust the parameters until the forward model of the "data" agrees with the "data". We have demonstrated the validity of the

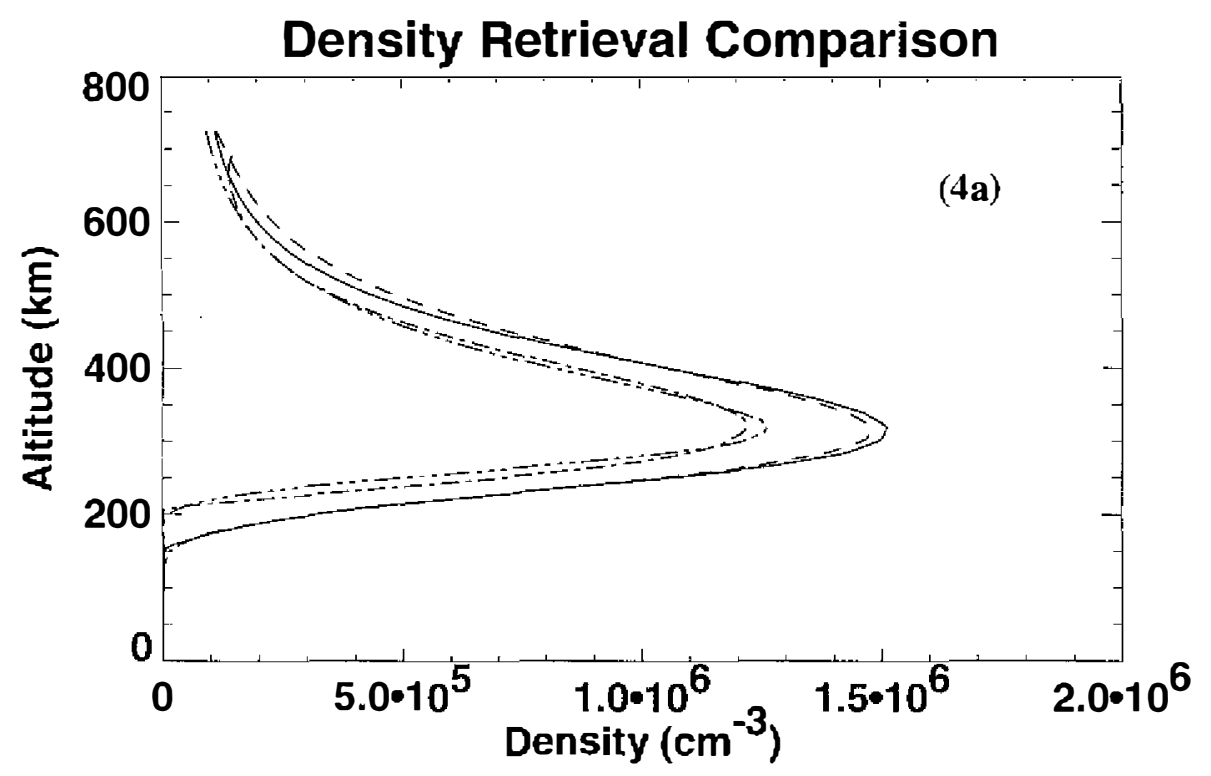

Fig. 4. Panel 4a shows a comparison of the IRI-90 density along the tangent ray trajectory (solid line) to: the two-dimensional algorithm fit (dashed line), the Abel inversion retrieval (dash-dot), and a discrete inverse theory fit assuming spherical symmetry and our modified Chapman layer parameterization (dash-triple dot). Both the Abel inversion and the discrete inverse theory inversion rely on the spherical symmetry assumption and are in error by approximately $20 \%$ near the peak while the two-dimensional inversion using the photometer "data" reproduces the "true" density very well. Panel 4b shows the fractional ((2D - IRI-90)/IRI-90) error between the two-dimensional retrieval and the IRI-90 ionosphere. In this panel, negative values denote that the two-dimensional retrieval is below the IRI-90 ionosphere. Panels $4 \mathrm{c}$ and $4 \mathrm{~d}$ show the IRI-90 ionosphere and the retrieved ionosphere, respectively. Note that the ionospheric peak height does not change with latitude in the retrieval, while it does in the IRI-90 ionosphere. The smooth curve rising from approximately $24^{\circ} \mathrm{N}$ in panel b represents the tangent point trajectory. 


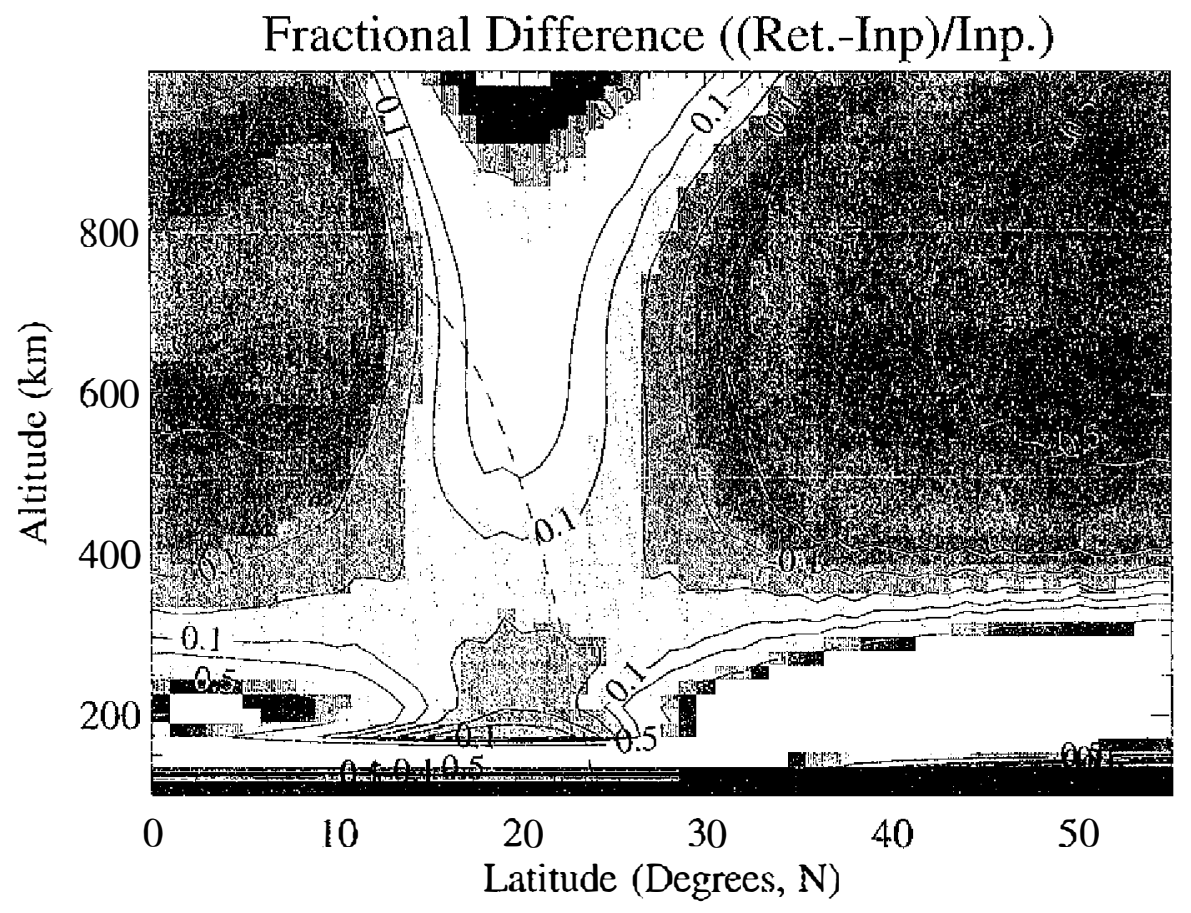

(4b)

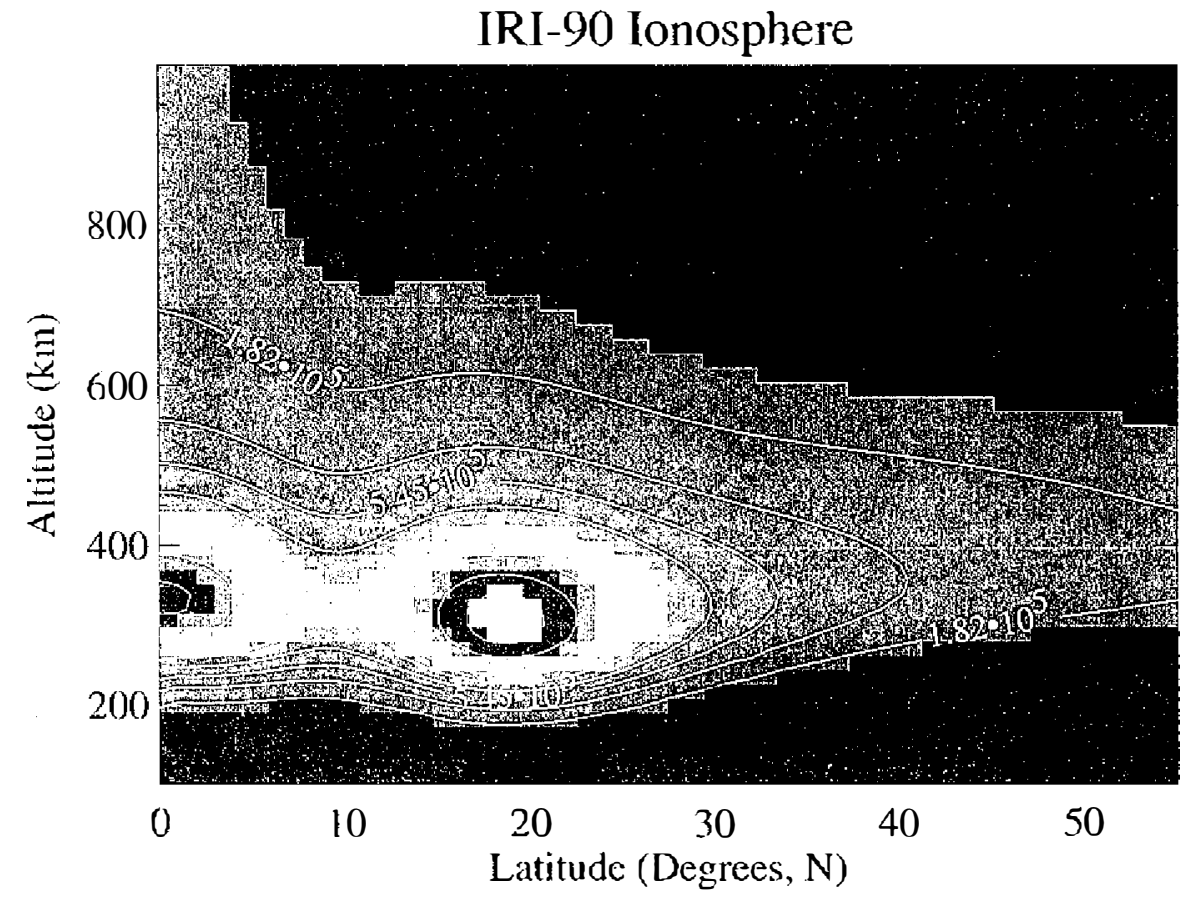

(4c)

(Fig. 4. continued.) 


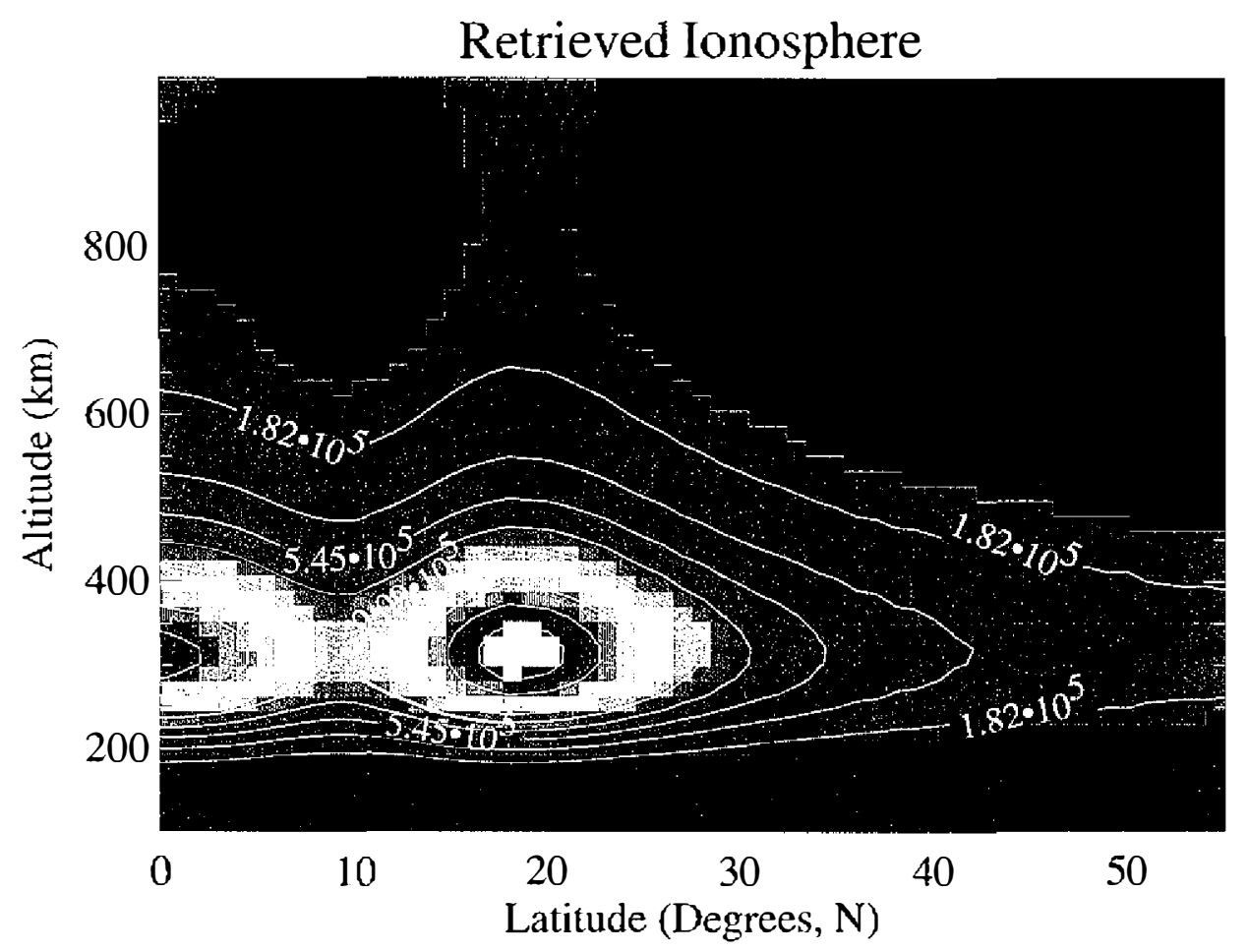

(4d)

(Fig. 4. continued)

algorithm in cases of high electron density gradients. The algorithm is based on Discrete Inverse Theory (Menke, 1989) and is flexible enough to permit the inclusion of a variety of ionospheric parameterizations.

Overall, the algorithm does a good job of retrieving the electron density from the GPS TEC's and the photometer radiances. The technique permits an accurate retrieval of the ionosphere over a wide range of latitude in areas with high gradients. The COSMIC constellation is expected to produce 4000 occultations per day, many of these will be near the orbit plane on the nightside where our algorithm works well.

Acknowledgments This work was supported by the COSMIC Program at the University Corporation for Atmospheric Research and by the Office of Naval Research. We are also grateful to the referee who reviewed this paper. The referee's comments substantially improved the quality of the paper.

\section{REFERENCES}

Abramowitz, M., and I. A. Stegun, 1972: Handbook of Mathematical Functions with Formulas, Graphs, and Mathematical Tables, Dover, Mineola, New York.

Anderson, D. E., Jr., and R. R. Meier, 1985: The O II 834 A Dayglow, A General Model for Excitation Rate and Intensity Calculations. Planet. Space Sci., 33, 1179-1186. 
Barth, C. A. and S. Schaffner, 1970: OGO 4 Spectrometer Measurements of the Tropical Ultraviolet Airglow. J. Geophys Res., 75, 4299-4306.

Bevington, P. R., 1969: Data Reduction and Error Analysisfor the Physical Sciences, McGrawHill, New York, NY.

Bilitza, D., 1990: International Reference Ionosphere 1990, National Space Science Data Center, NSSDC/WDC-A-R\&S 90-20, Greenbelt, Maryland.

Chakrabarti, S., R. Kimble, and S. Bowyer, 1984: Spectroscopy of the EUV (350-1400 A) Nightglow. J. Geophys. Res., 89, 5660-5664.

Chamberlain, J. W. and D. M. Hunten, 1987: Theory of Planetary Atmospheres: An Introduction to Their Physics and Chemistry, Academic Press, Inc., Orlando, FL..

Chandra, S., E. I. Reed, R. R. Meier, C. B. Opal, and G. T. Hicks, 1975: Remote Sensing of the Ionospheric F Layer by Use of O I 6300-A and O I 1356-A Observations. J. Geophys. Res., 80, 2327-2332.

Duderstadt, J. J., And W. R. Martin, 1979: Transport Theory, John Wiley and Sons, New York, NY.

Dymond, K. F., S. E. Thonnard, R. P. McCoy, and R. J. Thomas, 1996: A Technique for Determining F Region Electron Densities Using Optical Measurements of Recombination Radiation, Proceedings of the 1996 Ionospheric Effects Symposium, 7-9 May 1996, Alexandria, VA., 346-353.

Dymond, K. F., S. E. Thonnard, R. P. McCoy, and R. J. Thomas, 1997: An Optical Remote Sensing Technique for Determining Nighttime F Region Electron Density. Radio Science, 32, 1985-1996.

Feldman, P. D., A. F. Davidsen, W. P. Blair, C. W. Bowers, S. T. Durrance, G. A. Kriss, H. C. Ferguson, R. A. Kimble, and K. S. Long, 1992: The Spectrum of the Tropical Oxygen Nightglow Observed at 3 A Resolution With the Hopkins Ultraviolet Telescope. Geophys. Res. Lett., 19, 453-456.

Hajj, G. A., E. R. Ibanez-Meier, E. R. Kurzinski, and L. J. Romans, 1994: Imaging the Ionosphere with the Global Positioning System. Inter. J. Imaging System. Tech., 5, 174184.

Hedin, A. E., 1987: MSIS-86 Thermospheric Model. J. Geophys. Res., 92, 4649.

Hicks, G. T. and T. A. Chubb, 1970: Equatorial Aurora/Airglow in the Far Ultraviolet. $J$. Geophys. Res., 75, 6233-6248.

Meier, R. R., 1991: Ultraviolet Spectroscopy and remote Sensing of the Upper Atmosphere, Space Science Reviews, 58.

Melendez-Alvira, D. J., R. R. Meier, J. M. Picone, P. D. Feldman, and B. M. McLaughlin, 1999: Analysis of the Oxygen Nightglow. Measured by the Hopkins Ultraviolet Telescope: Implications for Ionospheric Partial Radiative Recombination Rate Coefficients. J. Geophys. Res., 104, 14901-14913.

Menke, W., 1989: Geophysical Data Analysis: Discrete Inverse Theory, International Geophysics Series, No. 45, Academic Press, San Diego.

Picone, J. M., R. R. Meier, O. Kelley, K. F. Dymond, R. J. Thomas, D. J. Melendez-Alvira, and R. P. McCoy, 1997: Investigation of Ionospheric $\mathrm{O}^{+}$Remote Sensing Using the 834-A Airglow. J. Geophys. Res., 102, 2441-2456. 
Press, W. H., B. P. Flannery. S. A. Teukolsky, and W. T. Vetterling, 1992: Numerical Recipes: The Art of Scientific Computing, Cambridge University Press, New York, NY.

Samson, J. A. R., 1967: Techniques of Vacuum Ultraviolet Spectroscopy, Pied Publications, Lincoln, Nebraska.

Strickland, D. J., and M. H. Rees, 1974: The O I 1304 and 1356 Emissions in Aurorae. Planet. Space Sci., 22, 465-481.

Strickland, D. J., 1979: The Transport of Resonance Radiation in a Non-Isothermal MediumEffect of Varying Doppler Width. J. Geophys. Res., 84, 5890-5896.

Tinsley, B. A., 1972: O I and N I Allowed Transitions in the Airglow and Aurora. Ann. Geophys., 28, 155-168.

Tinsley, B. A., A. B. Christensen, J. Bittencourt, H. Gouveia, P. D. Angreji, and H. Takahashi, 1973: Excitation of Oxygen Permitted Line Emissions in the Tropical Nightglow. $J$. Geophys. Res., 78, 1174-1186.

Tinsley, B. A., and J. A. Bittencourt, 1975: Determination of F Region Height and Peak Electron Density at Night Using Airglow Emissions from Atomic Oxygen. J. Geophys. Res., 80, 2333-2337.

Wang, J., D. G. McCoy, A. J. Blake, and L. Torop, 1987: Effects of the Close Approach of Potential Curves in Photoabsorption by Diatomic Molecules-II. Temperature Dependence of the O2 Cross Section in the Region 130-160 nm. J. Quantitative Spectroscopy and Radiative Transfer, 38, 19-27. 